\title{
EL PSICÓLOGO QUE SE BUSCA
}

\section{Helio Carpintero}

Universidad Complutense de Madrid

\section{RESUMEN}

La creación de un espacio europeo universitanio en la Union Europea ha obligado a revisar los curricula de las distintas enseñanzas, y reorganizar sus contenidos. En psicologia, hay que destacar los trabajos de la European Federation of Psychological Associations (EFPA), asi como los de la Conferencia de Decanos de Facultades de Psicología, de España, que vienen elaborando sendas propuestas de plan de formación.

En este contexto, el autor subraya la necesidad de conceder una mayor presencia a la formación práctica, sugiriendo la creación de un Centro de Psicología Aplicada unido a cada Facultad; la necesidad de una mayor formación científica en los estudiantes que aspiran a cursar esta carrera, y la conveniencia de establecer estudios optativos que permitan a los psicólogos clínicos que 10 deseen obtener licencia para prescribir tratamientos farmacológicos que apoyen sus terapias psicológicas.

Palabras clave: CURRÍCULO, FORMACIÓN DEL PSICOLLOGO, PSICOLO GIA ESPAÑOLA 


\section{SUMMARY}

The European Union has created a European educational Space, in which curricula and training plans have to maintain certain common traits. As a consequence, all its members have initiated a revision of curricula, to be ended in a few years. In the case of psychology, some interesting efforts have been made by EFPA and by the Spanish Conference of Deans of Psychology Faculties.

In such a context, some proposals are made in this article, concerning the psychologist's curriculum. It emphasizes the need of a strong practical training of students, the creation of a Applied Center in each Faculty, and the requirement of a high school training oniented toward natural science, in all those students entering these faculties. It is also suggested the opportunity of introducing as an option those matters that would enable the psychologist to prescribe pharmacological drugs which will enhance his psychological clinical treatments.

\section{Key words: CURRICULUM, PSYCHOLOGIST'S TRAINING, SPANISH} PSYCHOLOGY

La incorporación de España a la Unión Europea ha terido, entre otros efectos, el de su integración en un espacio educativo común, que ha forzado la revisión y progresiva homogenización de títulos académicos y profesionales. Con ello, se han puesto en cuestión muchas cosas: los contenidos de los curricula que se vienen impartiendo en facultades y escuelas técricas, su adecuación a las necesidades del mercado de trabajo, su duración, los modos efectivos de ejecución, sus posibilidades de equiparación de unos países a otros. Desde el entorno en que nos hallamos han venido, pues, los requerimientos para que se revisara la efectiva estructura de nuestra práctica formativa. El acuerdo de Bolonia de 1999, suscrito por los ministros responsables de educación de todos los países miembros, ha marcado una señal de 'no retorno' en este esfuerzo de revisión y convergencia. En el proceso se halla envuelta, como no podía ser de otro modo, la Psicología (Peiró y Lunt, 2002). 
La exigencia de un proceso de reflexión y análisis que oriente y dirija los movimientos de convergencia europea han venido estimulando a diversos colectivos y organismos más o menos directamente implicados en la tarea. A nadie puede sorprender que haya sido en el campo de la psicología del trabajo y las organizaciones donde va ya para tres lustros, se ha comenzado a examinar de modo técnico el problema. El European Network of Organizational and Work Psychologists realizó una labor seria y minuciosa, en un intento de diseñar el curriculum de su especialidad, aplicando a ello sus propios conocimientos de análisis de tareas, formación y entrenamiento en habilidades, y características de la demanda social hacia ese tipo de especialista (Roe et al., 1994; Roe, 2003, ENOP, 1995). Después de venir realizando este tipo de análisis con tantas y tan distintas profesiones y tan diversos puestos de trabajo, desde los primeros logros de la profesiografía y la orientación profesional de las primeras décadas del siglo $\mathrm{XX}$, era forzoso que al fin se terminara por aplicar, en un movimiento de reflexión, hacia la realidad propia de los psicólogos del trabajo.

Ese modelo organizacional por fuerza habia de influir en los trabajos e investigaciones que han seguido sus pasos. Es por lo menos forzoso referirse a dos líneas de análisis y revisión que en la actualidad están en marcha.

Uno es el proyecto de plan directivo común de los estudios de Psicología y el consiguiente Diploma Europeo de Psicología (en que vienen actualmente laborando un grupo de expertos colegas, yen donde está nuestro país admirablemente representado por el $\mathrm{Dr}$. J.M.Peiro, de la Universidad de Valencia) dentro del campo de aplicación de los Proyectos Leonardo, de la Unión Europea (www.europsych.org). El otro es el que, propuesto por la Conferencia de Decanos de Psicología sobre el curriculum a impartir en sus facultades, se ha presentado para evaluación de la Agencia Nacional de Evaluación (ANECA), y se halla también en fase de avanzado progreso (Pich y Conchillo, 2003).

Coinciden, así, en este momento, un estudio internacional, estrictamente europeo, sobre el "Psychology Training in Europe", o <EUROPSYT>, respaldado por la European Federation of Psychological Associations (EFPA) -que además está elaborando 
una propuesta de Diploma Europeo de Psicología <EUROPSY>-, y otro estrictamente nacional, impulsado por las autoridades académicas responsables, en la tarea de un reanálisis del campo de acción del psicólogo y la formación que éste exige, lo que representa un singular momento de toma de conciencia de la psicología, tanto académica como profesional, en el que toda experiencia, singular o de grupo, puede aportar alguna luz complementaria que enriquezca el resultado colectivo.

\section{EL MODELO DE ANÁLISIS}

En general, el modelo genérico de análisis a que se viene sometiendo el problema suele adoptar la estructura de una tabla de Input-output. De un lado, se tienen en cuenta las varias actividades y roles que los psicólogos vienen desempeñando de modo relativamente estable en nuestro tiempo (outputs); de otro, se ha de considerar los aprendizajes, conocimientos, habilidades, y actitudes que han de hacer posibles tales desempeños (inputs). En la práctica, estos análisis tienen como meta establecer las "competencias" propias del psicólogo. Se entiende por "competencia", en palabras de Roe, "una aptitud aprendida con la que ejecutar adecuadamente una tarea, una obligación o un rol" (Roe, 2002).

Se han señalado por algunos autores una serie de veinte competencias primarias, referidas a una serie de tareas que usualmente el psicólogo profesional ha de realizar cuando lleva a cabo una intervención concreta a instancias de un cliente que solicita su concurso. Con el fin de determinar la adquisición de esas competencias, el proyecto EUROPSYCH, antes mencionado las ha agrupado en seis categorías o roles profesionales: especificación de la intervención (goal specification); información diagnóstica (assessment) de los individuos o grupos con que se ha de trabajar; desarrollo técnico (development) con puesta a punto de los modelos e instrumentos para la intervención; intervención (intervention) propiamente dicha mediante la aplicación de metodologias y tecnologias existentes; evaluación de la intervención (evaluation), y, finalmente, comunicación de los resultados (Bartram \& Roe, 2004). 
Tal procedimiento analítico tiende, no obstante, a moverse en un plano de alta generalidad, que ha de concretarse en el desempeño profesional en cada uno de los ámbitos de especialización, y desde el cual no es particularmente fácil el descenso al nivel más cotidiano de la enseñanza. En todo caso, conviene notar que al adoptar aquel procedimiento u otro análogo posible, el supuesto básico aceptado implícitamente es que el psicólogo es un profesional formado mediante "un saber técnico capaz de precisar, diagnosticar y modificar el ajuste vital del individuo -0 del grupo- a su contexto situacional" (Carpintero, 2004), llevando a cabo la solución de problemas que afectan a tales individuos o grupos. También se asume que la finalidad que se pretende con su formación es, justamente, permitirle y capacitarle para ese ejercicio profesional.

Ahora bien, a mi juicio, y aceptando en su integridad los logros de los análisis profesiográficos a que por ese camino se llega, es preciso admitir que todos los modos de intervención que hayan de practicarse han de ir fundados en un conjunto de conocimientos a los que hay que caracterizar, grosso modo, como científicos (Peiró, 1994), pero la variedad de propuestas 'científicas' que circulan como tales en el mercado actual, y su combinación, frecuentemente sin orden ni método, con otras propuestas que tienden a definirse como 'humanistas', 'integrales', 'integradoras', etc. -en contradistinción con una supuesta 'ciencia impersonal deshumanizada'- han conducido a una situación confusa en campos importantes de la praxis, que es urgente abordar al menos de modo simultáneo al examen profesiográfico arriba mencionado. Me bastará aquí recordar unas palabras de tres reputados profesores de psicología clínica de nuestro ámbito universitario, indicativas del clima que todavía hoy reina en ciertos ámbitos:

"Resulta doloroso, o incluso patético, para los que somos profesores universitarios, escuchar a los alumnos de psicología decir que es importante conocer un poco de todo, para ver qué es más interesante o mejor." $Y$ añaden:

"Es difícil entender, y hacer entender a los alumnos, que la eficacia o la idoneidad de un conocimiento científico ... no puede depender de las impresiones personales al respecto" (Labrador, Echeburúa y Becoña, 2000). 
Con otras palabras, se trata de asentar la formación toda sobre una base que haga posible la adopción de una mentalidad cientifica. $Y$, con ella, la convicción de que el problema a considerar es esencialmente una perturbación o alteración de comportamientos personales, determinados por procesos mentales cognitivos y/o afectivos. Como decía M. Yela, la psicología se ocupa de "acciones significativas", esto es, de comportamientos dotados de una base orgánica, y ejecutados en un marco de entidades físicas, que al tiempo poseen 'sentido' 'significación' o 'valor' para uno o muchos sujetos que los realizan, los contemplan, los juzgan y padecen, interpretándolos y evaluándolos ya sea de un modo consciente $o$ inconsciente. $Y$ ante tales acciones cabe desde luego una aproximación científica.

\section{LA MENTALIZACIÓN DESEADA}

Recuerdo haber oido a S. Grisolia, notable investigador con una larga experiencia académica en los Estados Unidos, que durante años, las escuelas de medicina americanas recomendaron la lectura del libro de Cajal sobre Reglas y consejos sobre investigación científica, discurso leido por él en su ingreso en la R. Academia de Ciencias Exactas, Físicas y Naturales, en 1897. Y, posiblemente, deberíamos todavía imitar aquella práctica en nuestras facultades aun hoy día.

Leyendo e interpretando al gran investigador, se advierte cuánto le importa hacer del conocimiento una aventura activa, y no una repetición memorística de hechos y datos. Aparte otros motivos extrinsecos que animen al trabajo, Cajal propone que se fomente la "afición decidida hacia la originalidad, gusto por la investigación, y el deseo de sentir las fruiciones incomparables que lleva consigo el acto mismo de descubrir" (Cajal, 1940,85).

Dicho de otro modo: cree que la mentalidad de investigación ha de adquirirse mediarite el trabajo activo, personal, sobre cuestiones reales, enfrentándose con problemas reales, y no mediante memorizaciones impropias del nivel de formación intelectual en que pretendemos movernos. 
Quien tenga experiencia docente en ciencias y en humanidades, podrá fácilmente contrastar hasta qué punto es usual en aquellas el orientar la formación hacia actos de evaluación consistentes en solución de problemas y desarrollos racionales de cuestiones, y cuánta memorización y exposición repetitiva de apuntes y, en ocasión, de manuales se ofrece en las pruebas y exámenes de las humanidades.

Sé que la fórmula de J. Dewey "aprender haciendo" ('learning by doing), parece ya una frase clásica a la que es difícil sacar más punta. Pero entiendo que la formación mediante el ejercicio y el uso de la propia razón es el único camino hacia la adquisición de una mentalidad inquisitiva, dubitante, que demanda pruebas respecto de las tesis que examina, y que ha hecho la experiencia de lo que significa 'entender' y 'no entender', y de la diferencia que hay entre ambos extremos.

Sólo desde una enseñanza activa se adquiere el sentido y el valor del componente metodológico del saber, la relevancia del modo de hacer preguntas y formular los problemas, y el interés del razonamiento por analogia, que lleva a descubrir en modelos y planteamientos lejanos a las cuestiones que se tienen entre manos modos, relaciones y aplicaciones que son transferibles a la propia investigación.

Desde esa visión activa, crítica e independiente, que Cajal defendió incansablemente, es fácil llegar a ciertas conclusiones de orden práctico y de relativa aplicabilidad. Este tipo de formación exige el trabajo activo del alumno, la evaluación por tareas y trabajos personales resueltos, la esencial combinación de la clase magistral con los ejercicios de laboratorio, las lecturas prácticas, los comentarios de trabajos empíricos relevantes, $y$, en fin, el aprendizaje efectivo del manejo de los bancos de datos, de los portales especializados de Internet, y de la lectura de autores clásicos, en los que descubrir los modos de pensar y resolver dificultades, sin tratar de convertirlos en mero artículo de diccionario ni en objeto de veneración.

Lo que antecede, bien lo sé, se refiere al cómo más bien que al qué.

Pero es que el cómo delimita el ámbito de los contenidos, en cuanto estos han de ser susceptibles de cuestionamiento empírico, 
o de análisis conceptual riguroso, y es funcionalizando los contenidos y convirtiéndolos en problema como es posible que algo sea, efectivamente, objeto o contenido de un curso de formación universitaria que se ajuste a las condiciones propias del pensamiento crítico.

Se requiere, además, a mi juicio, de una cierta organización institucional sin la cual las pretendidas aspiraciones de cientificidad y rigor pueden quedar vacías de cumplimiento.

\section{REQUIRIENDO UNA ORGANIZACIÓN ADECUADA}

Consideraré tan solo algunos puntos que me parecen cruciales.

a) Ratio alumnos/profesor. La excusa que hemos aplicado en numerosas ocasiones a nuestra enseñanza defectuosa o limitada ha sido razonable: la masificación del número de alumnos ha impedido la enseñanza de calidad. Se optó por la cantidad. Hubo razones para ello. Pero todos sentimos que ha llegado la hora de producir el paso de la cantidad a la cualidad, como exigía la buena dialéctica.

Un primer paso es la transformación de la ratio profesor /alumno, hasta los niveles que hagan posible una continuada interacción entre ambos, y una supervisión de los ejercicios, redacciones y 'papers' que son necesarios para mantener fluida y activa la mente de los que se están formando.

b) Formación de base. Una segunda determinación que habría que adoptar es la implantación del requisito de haber cursado estudios secundarios de ciencias, para poder tener acceso a las facultades de psicología. La experiencia en nuestro país ha mostrado los grandes inconveniertes que introduce en un plan curricular la pretensión de alcanzar un nivel de conocimientos matemáticos (estadística) y biológicos (neuroanatomía y fisiología, bioquímica), desde una formación básicamente de letras, con carencia de los conocimientos básicos en el nivel previo a la universidad, y la consecuente transformación y descenso del rivel de las enseñanzas universitarias mencionadas.

c) Formación práctica. Hay, sobre todo, que alcanzar una enseñanza de calidad en el campo de las disciplinas que exigen de los 
alumnos la realización de prácticas. Unas, sin duda, son las que corresponden al lado práctico u operativo de las enseñanzas teóricas que se integran en el curriculum. La participación en experimentos, los trabajos de observación de campo, las aplicaciones de pruebas y de instrumentos de evaluación, los comentarios de trabajos experimentales y de textos clásicos, la realización de diseños y la resolución de problemas de psicometria, son algunas de las actividades que es preciso integrar de modo masivo, Hace algún tiempo, hablando en nombre de la Conferencia de Decanos de facultades de psicología, A. Blanco decía que esta formación aplicada "es nuestra asignatura pendiente" (Blanco, 1998) Es la cuestión del 'practicum', que requiere consideración aparte.

d) Formación en desarrollo y diseño de intervenciones profesionales. En el ejercicio profesional, con frecuencia el psicólogo no planifica sus intervenciones aplicando metodologias, tecnologias, estrategias o procedimientos que están previamente establecidos. Unas veces ha de adaptar esos procedimientos y técnicas a su caso; otras ha de introducir elementos nuevos que son la resultante de su propio diseño. Existe una amplia información sobre los procesos de "desarrollo" de metodologías, tecnologías y conocimientos relevantes que pueden resultar útiles en la formación de los psicólogos. Este planteamiento implica una concepción de la intervención profesional que va más allá de la mera aplicación de técnicas y herramientas, y que planifica aproximaciones más globales de la intervención profesional y más ajustadas a las demandas de los clientes. Si asumimos esta aproximación se verá claro que la formación en "desarrollo" es fundamental para que la competencia correspondiente, mencionada arriba, pueda ser adquirida y puesta en práctica por e] psicólogo. (Roe, 1989; Peiró,1994)

\section{LA FORMACIÓN MEDIANTE EL ‘PRACTICUM}

Gran parte de la actividad profesional del psicólogo consiste en estudio empirico de casos, individuales o de grupo, que implican recogida normalizada de datos, análisis de los mismos, diseño de una intervención con o sin instrumentación, y evaluación de la misma. 
Prácticamente en todos esos pasos, se requiere un saber hacer (know how), que no puede lograrse por medio de una formación libresca, sino que exige aprendizajes varios: de relación e interacción con sujetos, conocimiento de la aplicación e interpretación de datos resultantes de instrumentos (entrevistas, pruebas psicométricas, pruebas proyectivas, medición de habilidades con aparataje dispuesto al efecto), junto con conocimientos relativos al modo de informar y lograr la conformidad de los clientes con vistas a la adopción de un tratamiento o de unos modos de afrontar situaciones, etc. Todo ello impuso la necesidad de incorporar al proceso de formación un 'practicum', concebido como una etapa de formación esencialmente preprofesional y práctica, que se tiende a realizar bajo la forma de actuación supervisada por psicólogos profesionales, muy frecuentemente anejos al ámbito académico.

El resultado puede ser bueno; no entro a juzgar sus méritos. Por su lado positivo, hay datos que aseguran que un porcentaje no despreciable de jóvenes licenciados consiguen un primer contrato en empresas donde han estado realizando una estancia de prácticas.

Sin embargo, he de notar que toda esa formación práctica queda fuera de la acción directa del profesorado universitario, responsable de la educación técnica integral de los futuros profesionales.

Creo, por otra parte, que entre las prácticas que llamaré "de asignatura" y aquellas otras finales, que se cumplirian en centros, empresas o instituciones determinadas, hay un nivel intermedio de formación, que se refiere a la adquisición de competencias genéricas del psicólogo, sea cual sea la rama que luego elija, competencias que tienen que ver con la realización de entrevistas a niños y adultos, aplicación y corrección de pruebas en vista a una evaluación individual, iniciación en dinámica de grupos, realización de historiales de casos, observación de conducta, redacción de informes, etc. Todo ello deberia llevarse a cabo en una Unidad o Centro de Psicología Aplicada, orgánicamente vinculada a la respectiva Facultad. Alli los distintos departamentos - clínicos, profesionales, organizacionales - podrían impartir esa formación, que se centraría en aspectos generales de la interacción personal y grupal a que ha de hacer frente el psicólogo de modo usual en su vida profesional. 
Piénsese, para poner un modelo análogo, en la obligación ineludible que exige a las Facultades de Medicina contar con un hospital anejo, en que aprender a ejecutar los contenidos de la profesión médica bajo la supervisión de los profesores integrados en los propios departamentos universitarios. $O$, también, en la utilización de Escuelas anejas a las facultades de educación, para facilitar la formación práctica de los estudiantes dedicados al magisterio de ciclo básico.

Esos Centros o Unidades crearían el espacio donde el profesor universitario podría transmitir enseñanza práctica en cierto tipo de contextos reales, y las Facultades de Psicología podría ofrecer a la sociedad un servicio modélico de intervención, básicamente definido por las capacidades operativas de los departamentos que en ellas se integran.

La carencia de estas Unidades de Psicología Aplicada ha hecho que haya por lo general una distancia excesiva entre la formación pre-profesional y la docencia usual impartida en las Facultades, distancia que convendría revisar.

Una posibilidad que habría que explorar es la de la utilización de aquellos Centros de Psicología Aplicada, que se han ido creando en muchas de nuestras facultades, para prestar atención y servicio por lo general a la comunidad universitaria, y en ocasiones también al público. Podrían tomarse, tras redefinirlos, como espacio para concentrar esa formación práctica de actividad preprofesional especializada de que estamos hablando. En ellos cabe desde luego esa presencia directa de los departamentos responsables de las especialidades, $y$ en el inmediato futuro, de los posgrados que se implanten. Allí llegan, además, casos de estudio en contexto real que pueden ser analizados y examinados. Redimensionando esos centros, de adoptarse esta vía, ello podría suponer: "a) una mayor integración entre conocimientos teóricos y práctica profesional; b) mayor facilidad para contar con una supervisión multidisciplinar y especializada, y c) mayores posiblidades de observar y participar en investigaciones clínicas que se practican" (García Vera, 2004). $Y$, añado yo, las Facultades de Psicología incrementarian su imagen de centro operativo y útil a la sociedad, lo que no deja de tener su interés y su valor añadido. 
A mi modo de ver, al reivindicar el papel de la Facultad de Psicología en la formación preprofesional de sus estudiantes, rectificariamos la tendencia contraria, que lleva a dejar en manos ajenas la educación y entrenamiento de nuestros propios licenciados. Para mí, resulta por lo menos inquietante el modo como se ha tratado de resolver el caso de la formación de los psicólogos clínicos a través del recientemente implantado sistema de formación PIR. A cuenta de los beneficios que representa el contacto con pacientes y clientes de los servicios de salud mental, incluidos en los centros dependientes del sistema nacional de salud, se ha puesto en manos de otros profesionales, básicamente médicos, distinguidos y competentes pero situados fuera del ámbito profesional de la psicología, lo que resulta ser el grado más avanzado de formación de los mencionados especialistas. De hecho, se ha hecho ya desde algunos ángulos serias criticas al sistema PIR, especialmente en relación con la falta de formación teórica en psicoterapia, la poca atención al entrenamiento en relaciones terapéuticas, y la carencia de líneas de investigación en la mayoría de los centros que soportan la presencia rotativa de los psicólogos en formación (Virués et al. 2003). Eso, para no hablar del exiguo número de plazas que se vienen ofreciendo de hecho en el programa.

Desde mi punto de vista, se trata, en definitiva, de encontrar aquella organización que haga posible la efectiva formación preprofesional en el círculo de la propia facultad, y que reconozca a su profesorado el derecho y el deber a formar teórica y prácticamente a sus estudiantes, asumiendo de pleno derecho dentro de su dedicación esta formación práctica que tan esencial resulta en nuestro dominio.

\section{¿CUÁL ES EL ROL DEL PSICÓLOGO?}

La historia muestra que en el campo de la psicología, lo que empezó siendo un dominio teórico, el estudio de la mente, y básjcamente de la mente del hombre, vino luego a desarrollar una amplísima variedad de modos de aplicación e intervención, referidos a una gama casi inacabable de tipos de comportamiento que se 
modulan y singularizan por los contenidos efectivos que en ellos se actualizan. La situación es tal, que tal vez serla preciso "introducir el reconocimiento de la diversificación de contextos de práctica profesional desde el inicio del ejercicio profesional independiente y con anterioridad a la especialización" (Peiro, 2003).

De todos modos, desde hace ya cerca de un siglo viene habiendo unos grandes sectores de especialización, que han sido usualmente reconocidos como tales tanto en organizaciones académicas como en foros y reuniones profesionales. Me refiero a ese catálogo que incluye la psicología clínica, la del trabajo y las organizaciones, la educativa, la social aplicada o comunitaria, y la orientación propiamente dirigida hacia la investigación básica -sea el estudio experimental de procesos básicos, la psicobiología, o las cuestiones de teoria psicométrica, por mencionar algunas de las direcciones que aquella suele tomar-.

En el documento elaborado por un comité de especialistas, que fundamenta el plan general que podría servir para orientar los proyectos de formación del psicólogo en Europa, (Proyecto EuroPsyT), se ofrece esta imagen de la psicología actual:

"La Psicología es una ciencia central ya que toca todos y cada uno de los aspectos de la realidad humana. Los psicólogos profesionales trabajan en una gran variedad de ámbitos, incluyendo el clínico, el educacional, el organizacional, el jurídico, con el fin de dar respuesta a las cuestiones de la vida colectiva y aumentar el bienestar de las personas, como individuos, en grupos y en sistemas sociales" (Lunt et al., 2003).

Pues bien, entiendo que, en la presente remodelación curricular a que estamos dirigiéndonos, convendría explicitar la doble línea histórica que agrupa y ordena la inmensa variedad de los estudios y trabajos que en psicología se han venido realizando desde su constitución como ciencia independiente: la investigación básica, y la aplicada. No son dos categorías que puedan aspirar a alcanzar igual volumen. Las exigencias y demandas de la sociedad, en el terreno de las aplicaciones, no pueden encajarse dentro de las dimensiones, por fuerza reducidas, de la investigación básica. Pero la categoría del tamaño no decide de la última relevancia ni de la importancia de la investigación a realizar. Como escribió B. Houssay, 
el gran investigador y Premio Nobel argentino de fisiología, "sin la investigación científica pura, una universidad o un país está condenado a la inferioridad" (Houssay, 1960)

Entiéndase, pues, que considero indispensable mantener vivo y activo en toda facultad universitaria el espíritu de la investigación básica, con todos los recursos necesarios para que su acción pueda lograr resultados efectivos; otra cosa será cuáles y cuántas líneas de investigación podrán tener cabida en cada lugar. No sólo eso: como enseguida diré, confío en que avancemos en la dirección de integración de los resultados de los trabajos básicos sobre la práxis de los aplicados.

Ahora bien, la gran mayoría de estudiantes de psicología salen de nuestras aulas para entrar en un mercado de trabajo que reclama profesionales capaces de actuar con competencia y sobre fundamentos científicos rigurosos en las múltiples áreas en que la existencia humana se halla comprometida. $Y$ en este punto de competencia y capacitación, dejando a un lado otras cuestiones que tal vez tendrían aqui cabida, me referiré exclusivamente a un punto que me parece merecedor de toda nuestra atención. Me refiero a la, a mi juicio, necesaria pero todavía lejana capacitación del psicólogo para el empleo responsable de aquel apoyo farmacológico que se ha revelado como indispensable para consolidar formas de intervención psicológica cognitiva o conductual.

Entiendo, desde luego, que la praxis del psicólogo está abierta a múltiples formas de cooperación interdisciplinar. Lo está, desde luego, en todas sus formas de acción aplicada. Ha de cooperar con economistas, sociólogos y expertos en ciencias del trabajo en el mundo de las organizaciones y de las empresas; otro tanto ha de hacer con los educadores, los técnicos deportivos, los abogados, los jueces, los psiquiatras y los médicos generalistas, los técnicos de prisiones o los expertos en seguridad vial. Es el riesgo y es también el timbre de distinción de una ciencia que está interesada en lograr la adecuada adaptación del hombre a la casi infinita variedad de circunstancias en que éste puede hallarse.

Ahora bien, en el campo de la intervención clínica, en innumerables ocasiones, el psicólogo se ve obligado a sostener y apoyar su acción específica con la incorporación de ciertos fármacos, cuya 
acción psicotrópica viene a reforzar las medidas conductuales aplicables al caso. Quien quiere un fin que le es propio, ha de poder poner los medios para ello; de otro modo, estaría subaltemado, subordinado a otro técnico, que podría impedirle el cumplimiento de su tarea. Desde los años 80, en Estados Unidos se ha iniciado un movimiento que trata de conseguir para el psicólogo clínico la capacidad de prescribir ciertos fármacos de uso habitual en su combinación con ciertos tratamientos. Desde hace más de una década, se ha hablado del tema como de una "nueva frontera" (De León et al., 1991); y se han dado ya pasos de interés, en la dirección deseada, de modo que en algunos (pocos) estados se ha logrado esa licencia, gracias, desde luego, al establecimiento de unos planes formativos muy amplios y rigurosos en bioquímica y farmacologia que han de ser superados antes de obtener el permiso mencionado.

El asunto, antes o después, habremos de abordarlo. Convendría tenerlo bien presente desde ya en el horizonte de nuestro próximo curriculum.

\section{CIENCIA BÁSICA Y CIENCIA APLICADA}

La segunda cuestión que quisiera considerar aquí se refiere a la necesidad de generalizar la demanda de que las técnicas que se introduzcan en los programas de formación de profesionales estén de un modo $u$ otro referenciados en el marco de los procedimientos que han sido retrendados por la evidencia científica.

Recuérdense las palabras de Eysenck en su famoso estudio sobre los efectos de la psicoterapia, tan combatido al tiempo que oportuno: "En ausencia de un acuerdo entre hechos y convicciones, hay una urgente necesidad de que disminuya la fuerza de la convicción y aumente el número de los hechos de que quepa disponer" (Eysenck, 1952/1973). El problema, naturalmente, estaría resuelto si todos nuestros profesionales asumieran una mentalidad sistemática, o científica si se prefiere, que llevara a depositar su convicción en aquellos conocimientos o procedimientos para los que hubiera hechos probatorios adecuados. Pero, desde los tiempos de Sócrates, vienen en el mundo de la cultura occidental combatiendo entre sí 
los sofistas que se mueven en el orden del parecer, y los que teorizan en busca de la verdad.

Puede además ocurrir de hecho que no haya en todo el asunto el menor rastro de mala voluntad. Como han escrito unos expertos muy solventes, "por mucho que se haya avanzado en los tratamientos psicológicos, lo que parece ser hoy eficaz - la práctica psicológica basada en la evidencia- dista de estar disponible para la mayoría de la población, en parte porque algunos tratamientos 'eficaces' en la investigación no se han mostrado tan 'efectivos' en la práctica clínica, y en parte porque muchos profesionales no están suficientemente formados en estas técnicas" (Labrador, et al., 2000, 303). Y si bien la deontología profesional de un lado, $y$, de otra parte, la creciente jurisprudencia en torno a los casos de 'malpractice', van imponiendo la necesidad de utilizar procedimientos que estén sólidamente fundados en datos de investigación previa que aseguren la corrección de su uso, tal vez no ha llegado aún el momento en que todos los profesores de psicología introduzcamos en el torso general de nuestras particulares enseñanzas esta necesidad de actuar sobre evidencias racionales y pruebas empíricas. Aunque entiendo que todavía suenan a muchos estas conclusiones como excesivas o dogmáticas, me parece que los investigadores y enseñantes que trabajamos en el campo de la psicologia científica habriamos de repetir y acomodar a nuestro propio terreno, generalizándola, esta afirmación de unos metodólogos que han venido trabajando en el campo del metaanálisis y la evaluación de resultados: "Sólo hay una Psicología Clínica aceptable, aquella que se sostiene sobre los principios del método científico" (Pascual, et al, 2004).

Ahora bien, esto nos lleva, precisamente, al núcleo mismo de la cooperación entre ciencia básica y ciencia aplicada. Desde los años 90, se han puesto en marcha en Estados Unidos, luego en el Reino Unido, $y$ en otros países sucesivamente, grupos de trabajo interesados en la detección de los Tratamientos con Apoyo Empírico, con aplicación de criterios rigurosos en su calificación. Tales estudios han puesto de relieve que "cada grupo de trastornos suele tener varios TAE (Tratamientos con Apoyo Empírico), con distintos o iguales grados de validez", y una gran parte de esos procedimientos son precisamente tratamientos basados en el modelo teórico de la te- 
rapia de conducta, lo que "avala la estrecha interacción entre la investigación básica y la tecnología derivada de ellas" (Mustaca, 2004). Una investigación básica, no habrá que decirlo siquiera, que en buena medida se ha hecho y se sigue haciendo gracias a trabajos llevadas a cabo en el campo de la conducta animal.

No sólo en clínica: en general, en psicología, "estamos... enfrentados al conocimiento de... 'estados mentales', vividos por un sujeto, cuya índole ha de ser interpretada por el psicólogo,... y ha de hacerse en términos de cualidades observables, datos orgánicos y vivencias subjetivas declaradas, que requieren una ulterior construcción conceptual por el profesional, que ha de realizar así un efectivo 'diagnóstico' de lo vivido por el sujeto;... proceso diagnóstico... que estará fundado en unas evaluaciones cuantitativas y descripciones cualitativas estructuradas, mediante las cuales podrá referirse a sistemas categoriales definidos que ordenan la multiplicidad aparentemente inagotable de vivencias que forman la vida mental" (Carpintero, 2002). Se trata, en suma, de que la integración de las esferas básica y aplicada, en psicología, ha venido siendo y posiblemente seguirá siendo la condición de la fundamentación y evidencia empírica última de sus procedimientos y aplicaciones, refrendando una vez más el alcance de la conocida sentencia de K. Lewin, sobre que "nada hay más práctico que una buena teoría".

\section{CONCLUSIÓN}

Llegados a este punto, entiendo que tal vez no he respondido exactamente a lo que la demanda cordial de mi amigo el Dr. Pelechano podía significar ('qué debe enseñarse a un psicólogo, qué debe saber un profesor de psicología'). Pero creo que hoy hay ya multitud de trabajos serios y meditados que han ido desbrozando ese terreno, a algunos de los cuales ya me he referido más arriba.

Entiendo, sin embargo, que el más grave problema que tenemos en nuestro caso, no sólo en nuestro país sino en otros muchos, es el de consolidar de una vez por todas la condición científica de nuestro campo disciplinar, y ajustar nuestra formación de profesionales al modelo generalizado de aprendizaje activo, en que se sustituya el memorismo por las evidencias, algo que en campos 
próximos -la medicina, la química, la ciencia natural-, ha conducido a una formación empírica, de amplia base experimental, donde $-y$ esta es la exigencia de nuestra singularidad- nos hemos de basar no solo en el laboratorio, sino en el trabajo de campo, y en el estudio contextualizado social, de gran parte de nuestros problemas. La naturaleza bio-social del hombre no se deja encerrar en matraces, ciertamente, pero la ciencia experimental de nuestros dias sabe ya cómo aproximarse al mundo social con rigor, con medida, con modelos conceptuales definidos, y con la pretensión, sobre todo, de tener pruebas o fundamentos tanto de lo que al final se afirme como de aquella intervención que se proponga.

Es esta 'Gestalt' general de nuestra formación la que me parece que necesita todavía más estudio, más ensayo (Hayes, 1996), y sobre todo, más aplicación en nuestras instituciones. Tal vez sus requerimientos no se cumplan porque haya limitaciones económicas, dificultades burocráticas del profesorado, o carencia de un plan global de formación profesional. Pero es hora de que reclamemos para el psicólogo la mejor formación posible, ya que en la hora terrible de la catástrofe, o en la más cotidiana de la familia, el hogar, la escuela - la empresa, surgen unos problemas conductuales y mentales, ante los que el hombre de nuestro tiempo tiene derecho a recibir el mejor, más probado y más satisfactorio tratamiento de entre los que la psicología de hoy ya conoce, y puede y debe proporcionar.

\section{BIBLIOGRAFIA}

Bartram, D. \& Roe, R. (2004) Definition and assessment of competences in the context of the European Diploma in Psychology, Msc. To appear in The European Psychologists, (en prensa).

Blanco, A. (1998) Requisitos y necesidades de formación para la psicología del siglo XXI, Revista de Psicologla General y Aplicada, 51(1):149-172.

Carpintero, H. (2002) Del diagnóstico a la evaluación. Una cuestión a debate, Acción psicológica, 1(3): 233-244.

Carplntero, H. (2004) Psicologia, comportamiento y salud. El lugar de la psicología en los campos del conocimiento, Infocop, num.extr.: 93-101.

DeLeon, P.H., Fox, R., Graham, S. (1991) Prescriptions privileges. Psychology's Next Frontier? American Psychologist, 46(4): 384-393. 
ENOP (1995). Reference Model for a European Curriculum in W\&O Psychology. Revised Version. European Network of Organizational and Work Psychologists Reports, N. 2. Maison des Sciences de l'Homme. Paris. (www.ucm.es/info/Psyap/enop/rmodel.html).

Eysenck, H.J., (1973) The effects of psychotherapy: an evaluation (orig.1952), in Eysenck, $\mathrm{HI} \&$ Wilson, G., eds., The experimental study of Freudian theories, London, Methuen, 365-384.

García-Vera, MP. (2004) Los centros universitarios de asistencia psicologica como centros de formación de posgrado en psicología clínica y psicoterapia: la unidad de psicología clínica y de la salud de la UCM, Papeles del psicólogo, 87:59-66.

Hayes, N. (1996) What makes a Psychology Graduate distinctive?, European Psychol., 1(2): 130-134.

Houssay, B. (1960) La investigación cientifica, 2 ed., Bs. Aires, Columba. Labrador, F., Echeburua, E., y Becoña, E., (2000) Guia para la elección de tratamientos psicológicos efectivos, Madrid, Dykinson

Lunt, I. et al. (2003) Una propuesta marco para la eduacion y la formación del psicólogo en Europa, [Proyecto EuroPsyT], Infocop, 19, 65-76 (orig. EFPA, 2001: http://www.europsych.org/).

Mustaca, A. (2004) Tratamientos psicológicos eficaces y ciencia básica, Revista Latinoamericana de Psicologia, 36 (1): 11-20

Pascual, J., Frlas, MD., Monterde, H., (2004) Tratamientos psicológicos con apoyo empírico y práctica clínica basada en la evidencia, Papeles del Psicologo, 87: 1-8.

Peiró, J.M. (1994) Intenvención Psicosocial en Psicología del trabajo y de las Organizaciones. En J.M. Peiró y J. Ramos (Dirs.): Intervención Psicosocial en las Organizaciones. Barcelona, PPU, pp. 3-65.

Peiró, JM. (2003) La enseñanza de la psicología en Europa. Un proyecto de titulación europea, Papeles del Psicólogo, 86: 25-33.

Peiró, J.M. Y Lunt, I. (2002) The context for a European Framework for Psychologists' Training. European Psychologist, 7, 3, 169-179.

Pich, J. y Conchillo, A. (2003) Las Facultades de Psicología ante el reto de la Convergencia Europea, Infocop, 19: 77-83.

Ramón y Cajal, S. (1940) Reglas y consejos sabre investigación cientifica, Madrid, Beltrán.

Roe, R. (2003) ¿Qué hace competente a un psicólogo?, Papeles del Psicólogo, 86: 1-12.

Virués J., Santolaya, F., Garcla Cueto, E. y Buela-Casal, G., (2003) Estado actual de la formación PIR: actividad clínica y docente de residentes y tutores, Papeles del psicólogo, 85: 37-47. 